Supporting Information

\title{
Composable Microfluidic Plates (cPlate): A Simple and Scalable Fluid Manipulation System for Multiplexed Enzyme- Linked Immunosorbent Assay (ELISA)
}

Ziyi He ${ }^{\mathrm{a}, \mathrm{e}}$, Justin Huffman ${ }^{\mathrm{a}}$, Kathrine Curtin ${ }^{\mathrm{b}}$, Krista L. Garner ${ }^{\mathrm{c}, \mathrm{d}}$, Elizabeth C. Bowdridge ${ }^{\mathrm{c}, \mathrm{d}}$, Xiaojun $\mathrm{Li}^{\mathrm{a}}$, Timothy R. Nurkiewicz ${ }^{\mathrm{c}, \mathrm{d}}$, and Peng $\mathrm{Li}^{\mathrm{a}, *}$

${ }^{a}$ C. Eugene Bennett Department of Chemistry, West Virginia University, Morgantown, WV 26506, USA.

${ }^{\mathrm{b}}$ Department of Mechanical and Aerospace Engineering, West Virginia University, Morgantown, WV 26506, USA.

${ }^{c}$ Department of Physiology and Pharmacology, West Virginia University, Morgantown, WV 26506, USA.

${ }^{d}$ Center for Inhalation Toxicology, West Virginia University, Morgantown, WV 26506, USA.

${ }^{\text {e }}$ Present address: College of Animal Sciences and Technology, Huazhong Agricultural University, Wuhan, Hubei 430070, China.

*Corresponding author: peng.li@mail.wvu.edu 


\section{Table of Contents}

Figure S1. The design and dimensions of the cPlate system.

Figure S2. Loading patterns of cPlate validation and parameter optimization in single biomarker analysis.

Figure S3. Performance of un-coated/patterned well plates and coated/patterned well plates after reagent loading and plate assembly.

Figure S4. Spatially resolved fluid mixing by plate assembly and volume consistency after washing.

Figure S5. Response curves, LODs, and dynamic ranges of activin, CRP and VEGF.

Figure S6. Linear correlations between cPlate ELISA and 96-well plate ELISA for activin and VEGF.

Figure S7. Comparison between conventional 96-well plate ELISA and the cPlate ELISA.

Experimental Details: Materials and Reagents, Device Design and Fabrication, Validation of the Loading, Incubation and Washing Process, Single Biomarker Analysis, Multiplexed Biomarker Analysis, Rat plasma analysis. 
a

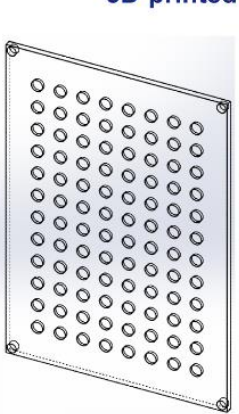

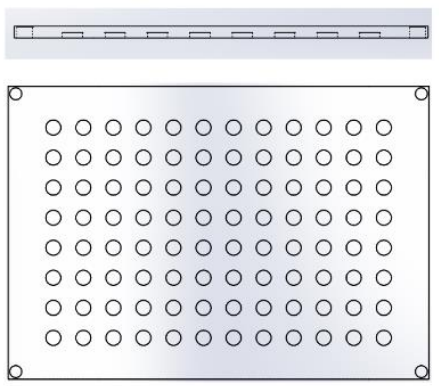

000000000000

b

3D-printed well plate for samples and reagents
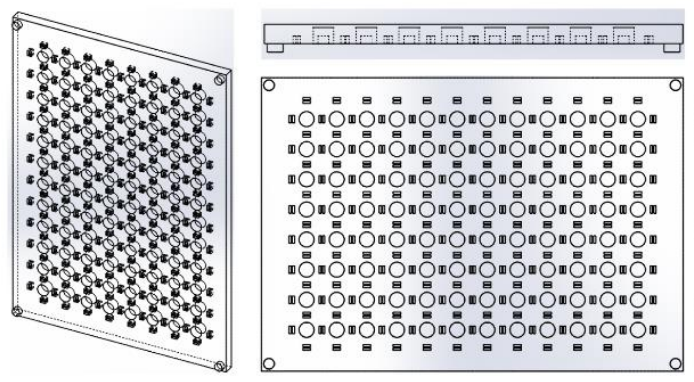

Well radius: $1 \mathrm{~mm}$

Well height: $0.5 \mathrm{~mm}$

Volume: $\sim 1.6 \mu \mathrm{L}$

C

Well plate shield

for superhydrophobic coating

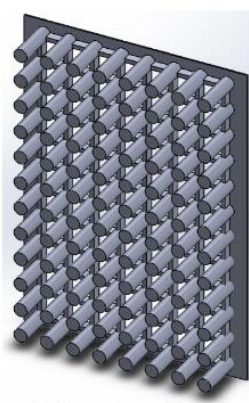

Pillar radius: $1 \mathrm{~mm}$ Pillar height: $7 \mathrm{~mm}$

d

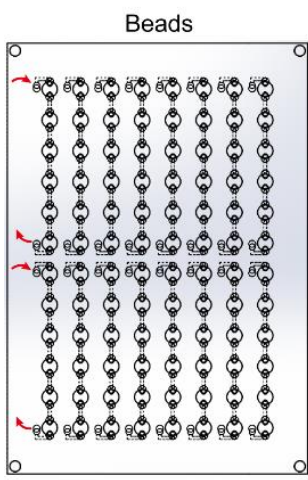

3D-printed channel plates for loading

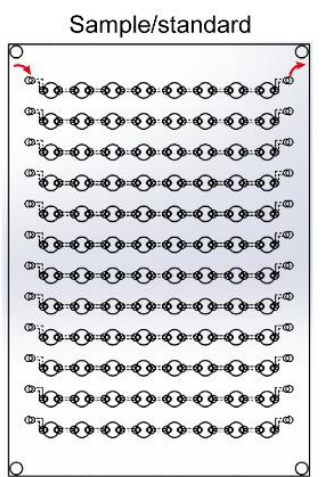

Well radius: $1 \mathrm{~mm}$

Well height: $1.5 \mathrm{~mm}$ (sample/standards, detection $\mathrm{Ab}$ ) $0.75 \mathrm{~mm}$ (HRP substrate)

Volume: $\sim 4.7 \mu \mathrm{L}$ (sample/standards, detection $\mathrm{Ab}$ ) $\sim 2.4 \mu \mathrm{L}$ (HRP substrate)

Small groove structures among wells: width: $0.3 \mathrm{~mm}$ length: $1 \mathrm{~mm}$ height: $0.75 \mathrm{~mm}$

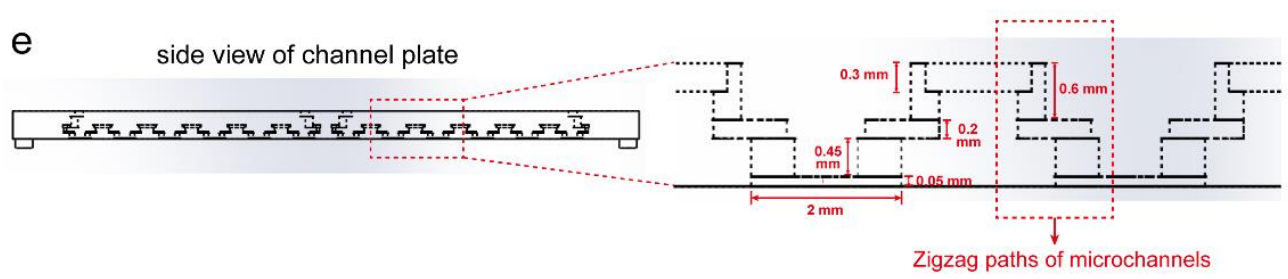

Figure S1. The design and dimensions of the cPlate system. (a) Design of 3D-printed magnetic bead well plate;

(b) Design of 3D-printed well plates for samples and reagents; (c) Design of well plate shield used in superhydrophobic coating; (d) Design of 3D-printed channel plates for reagent loading (red arrows indicate the reagent loading directions); (e) Side view of the design of the channel plates. 

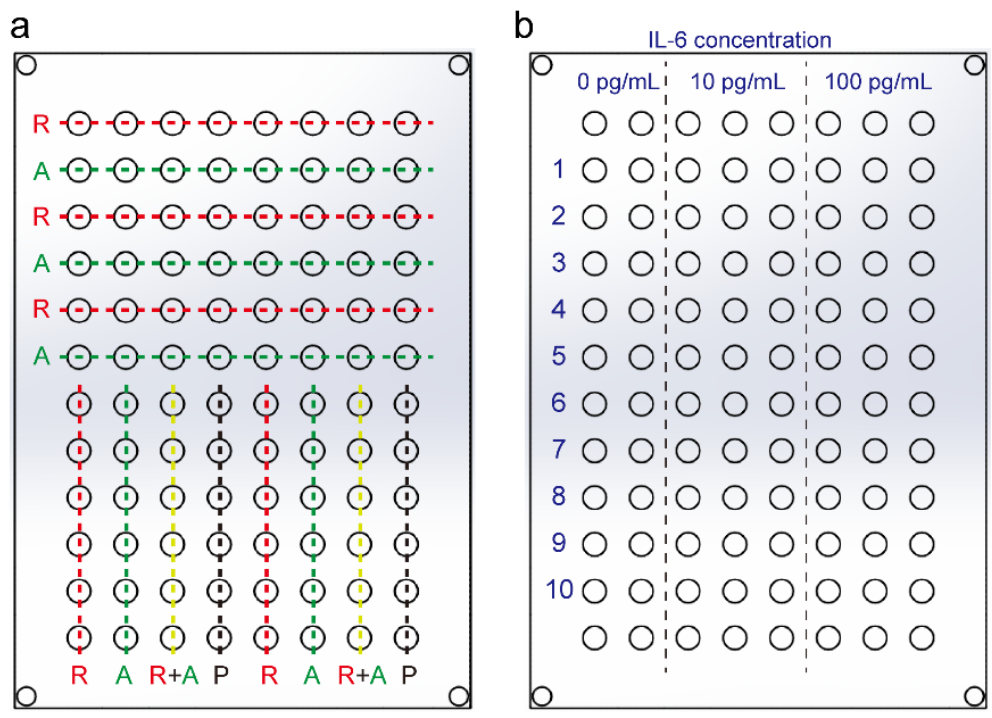

$\begin{array}{ccc}\begin{array}{c}\text { Capture Ab } \\ \text { concentration } \\ (\mathrm{mg} / \mathrm{mL})\end{array} & \begin{array}{c}\text { Magnetic beads } \\ \text { concentration } \\ (\mathrm{wt} \%)\end{array} & \begin{array}{c}\text { Detection Ab and } \\ \text { SA-HRP concentration } \\ (\mu \mathrm{g} / \mathrm{mL})\end{array} \\ 0.1 & 0.25 & 0.5 / 0.5 \\ 0.1 & 0.1 & 0.5 / 0.5 \\ 0.1 & 0.05 & 0.5 / 0.5 \\ 0.1 & 0.025 & 0.5 / 0.5 \\ 0.05 & 0.05 & 0.5 / 0.5 \\ 0.2 & 0.05 & 0.5 / 0.5 \\ 0.1 & 0.05 & 2 / 2 \\ 0.1 & 0.05 & 1 / 1 \\ 0.1 & 0.05 & 0.5 / 0.5 \\ 0.1 & 0.05 & 0.2 / 0.2 \\ & & \end{array}$

R: resorufin A: Alexa 488-strepavidin P: PBS

Figure S2. Loading patterns of cPlate validation and parameter optimization in single biomarker analysis. (a) Loading paths of resorufin, Alexa 488-strepavidin, and PBS for volume consistency validation; (b) Loading patterns of reagents in single biomarker analysis for parameter optimization. 


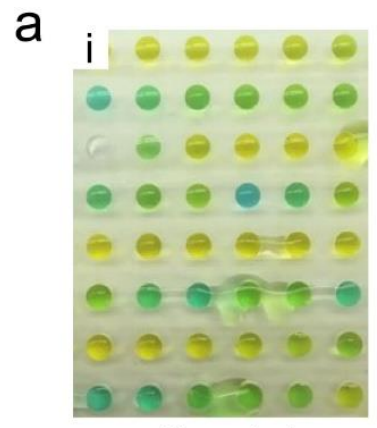

Uncoated

No groove structure

b

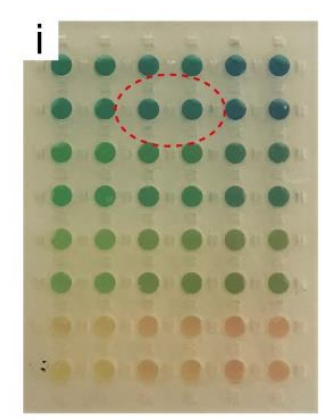

Coated

Groove structures

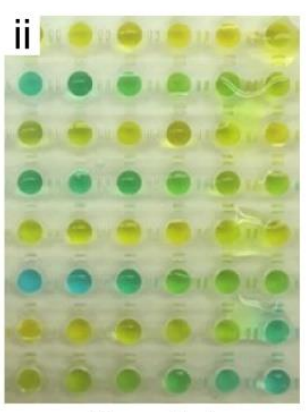

Uncoated

Groove structures

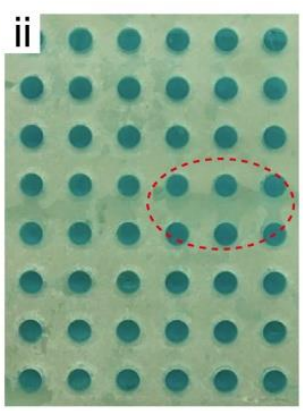

Coated

groove structure

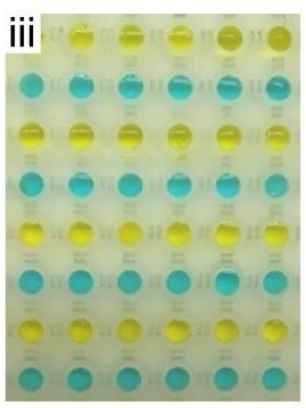

Coated

Groove structures

Figure S3. Performance of un-coated/patterned well plates and coated/patterned well plates after (a) reagent loading and (b) plate assembly. Without superhydrophobic coating, significant cross-contaminations among wells were observed due to fluid leakage during the loading process, and small groove structures were not able to prevent the leakage; while with superhydrophobic coating, different reagents were confined in their respective fluid paths, forming a clear pattern. For plate assembly, extra liquids squeezed out from wells after plate combination were held by the small groove structures among wells (Figure S3b-i, in red circle), which isolated independent reaction compartments; while without the structures, extra liquids spread between plates, which connected multiple reaction chambers and led to cross-talk among wells (Figure S3b-ii, in red circle). 
a

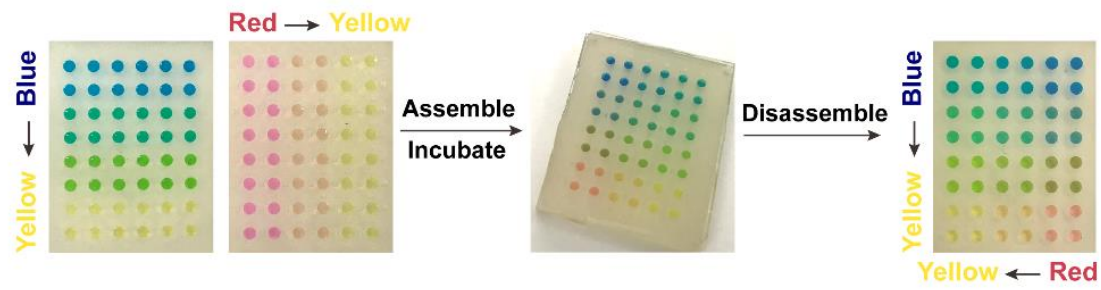

b
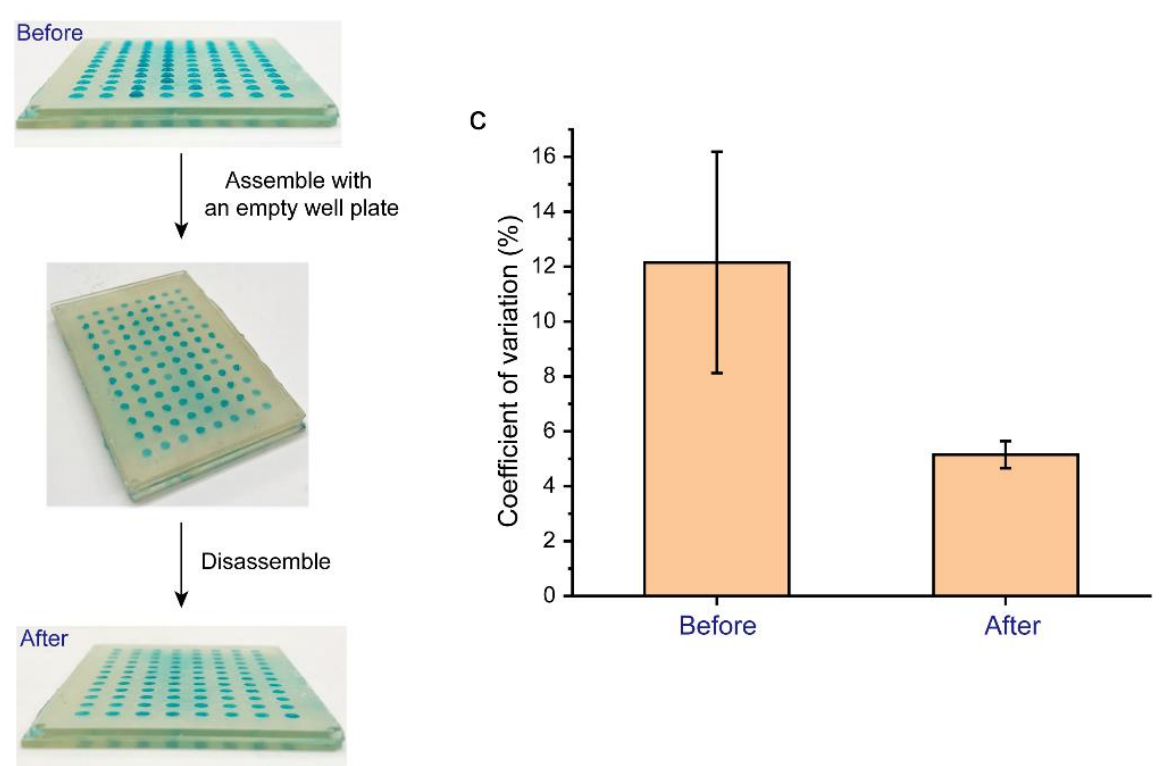

Figure S4. (a) Spatially resolved fluid mixing demonstrated by combining two plates respectively loaded with vertical and horizontal color gradient; (b) Images of well plate before and after assembling with an empty well plate (well height of $0.25 \mathrm{~mm}$ and well volume of $0.8 \mu \mathrm{L}$ ) after washing. After pouring out the washing buffer, the volume of liquids left in wells were always not uniform, some of which exceeded the volume of wells (1.6 $\mu \mathrm{L}$ ). When putting the empty plate onto the well plate, because of the hydrophilic inner well surface and superhydrophobic outer surface, extra liquid in each well filled into its corresponding empty well. When disassembling the two plates, shear force and surface tension would facilitate to disconnect the liquid in two corresponding wells, leaving a uniform volume distribution across the well plate; (c) Coefficient of variations (CV) of solution volumes in different wells across the whole plate before and after assembling with an empty well plate. The CVs are calculated based on the fluorescence intensity of solution in each well. 


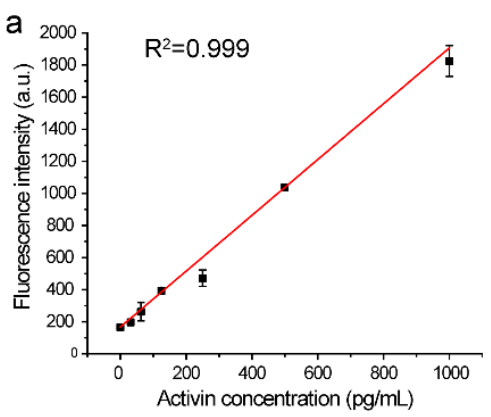

Dynamic range: $31.3-1000 \mathrm{pg} / \mathrm{mL}$ LOD: $7 \mathrm{pg} / \mathrm{mL}$

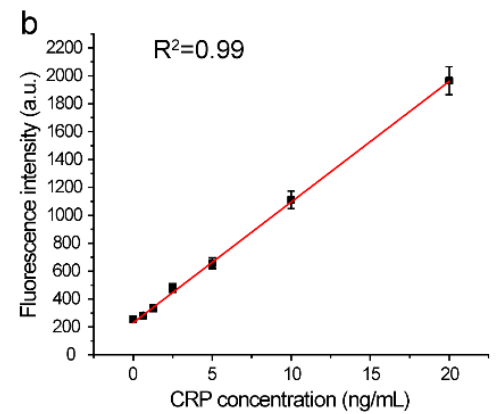

Dynamic range: $0.625-20 \mathrm{ng} / \mathrm{mL}$ LOD: $0.19 \mathrm{ng} / \mathrm{mL}$

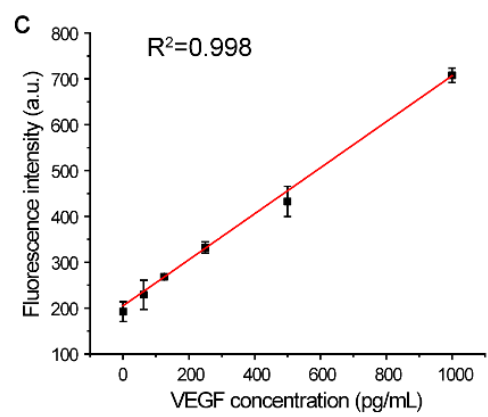

Dynamic range: $62.5-1000 \mathrm{pg} / \mathrm{mL}$ LOD: $13.4 \mathrm{pg} / \mathrm{mL}$

Figure S5. Response curves, LODs, and dynamic ranges of (a) activin, (b) CRP, and (c) VEGF. 
a

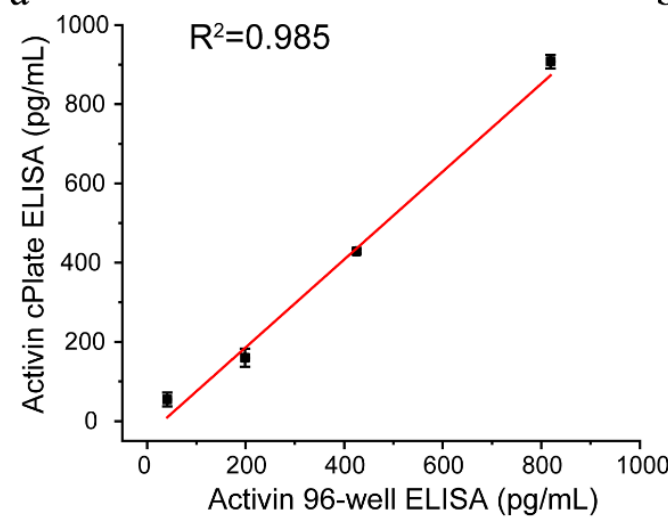

b

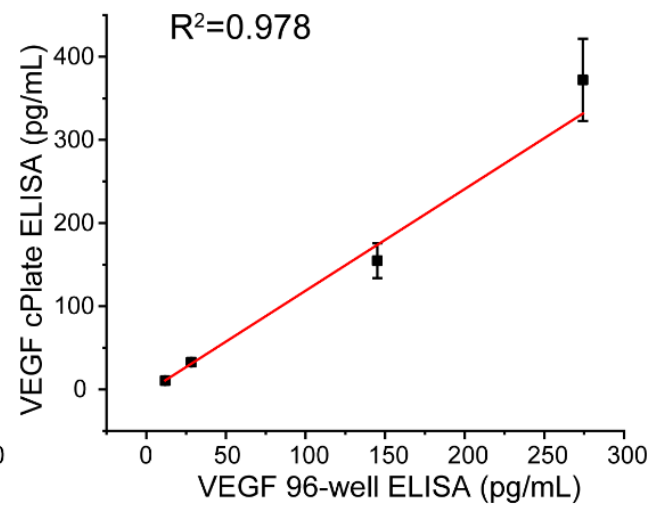

Figure S6. Linear correlations between cPlate ELISA and 96-well plate ELISA for (a) activin, (b) VEGF. 


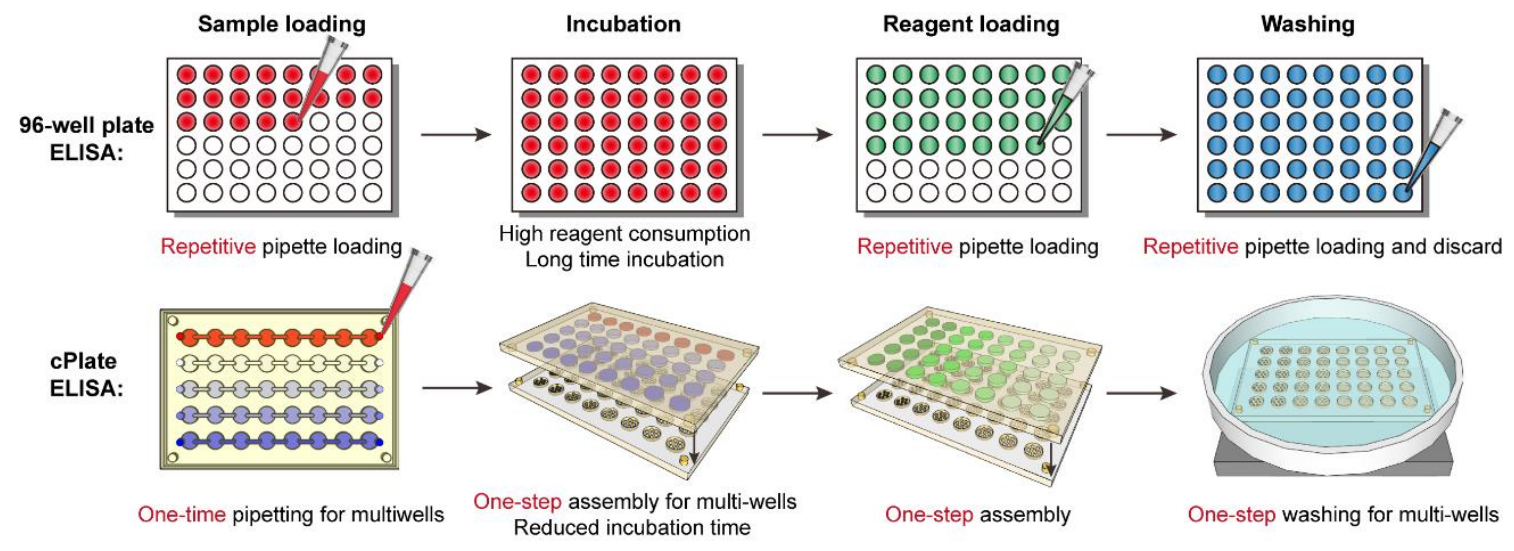

Figure S7. Comparison between conventional 96-well plate ELISA and the cPlate ELISA. Sample loading, incubation, reagent loading and washing steps are compared between the two platforms, and the 3D-printed cPlate system demonstrates superior advantage in all of the steps. For better visualization, this schematic is not drawn to scale. The actual size of a conventional 96-well plate is much larger than the cPlate. 


\section{EXPERIMENTAL DETAILS}

Materials and Reagents. Poly(ethylene glycol) diacrylate (PEGDA, MW 250), phenylbis (2,4,6trimethylbenzoyl) phosphine oxide (Irgacure 819) and fluorescein were purchased from Sigma-Aldrich (St. Louis, MO, USA). 2-nitrophenyl phenyl sulfide (NPS) was purchased from TCI (Tokyo, Japan). Ultra-Ever Dry superhydrophobic coating was purchased from Ultratech (San Jose, CA, USA). Epoxy glue (5-minute epoxy, Devcon) was purchased from ITW (Glenview, IL, USA). Food color was purchased from Wilton Industries. Polystyrene magnetic beads $(2.9 \mu \mathrm{m})$ were purchased from Spherotech (Lake Forest, IL, USA). NHS-activated magnetic beads $(1 \mu \mathrm{m})$, Streptavidin-HRP (SA-HRP) and QuantaRedTM enhanced chemifluorescent HRP substrate (Amplex Red) were purchased from Thermo Scientific (Waltham, MA, USA). 2,2'-azino-bis(3-ethylbenzothiazoline-6-sulphonic acid) (ABTS) was purchased from Alfa Aesar (Haverhill, MA, USA). Hydrogen peroxide (30\%, w/w) was purchased from LabChem Inc. (Zelienople, PA, USA). Isopropanol (IPA), bovine serum albumin (BSA) and Tween-20 were purchased from Fisher Scientific (Pittsburgh, PA, USA). Phosphate-buffered saline (PBS, pH 7.4) was purchased from Corning Inc. (Corning, NY, USA). General Block buffer was purchased from ImmunoChemistry (Bloomington, MN, USA). ChonBlock blocking/sample dilution ELISA buffer was purchased from Chondrex (Redmond, WA, USA). Human CEACAM1 matched antibody pair kit, anti-prostate specific antigen (PSA) antibody [8A6] and anti-PSA specific antibody [5A6] (biotin) were purchased from Abcam (Cambridge, UK). PSA (human seminal fluid) was purchased from Millipore (Burlington, MA, USA). IL-6 monoclonal antibody (5IL6), IL-6 monoclonal antibody (7IL6) (Biotin), human IL-6 lyophilized standard, CRP monoclonal antibody (C5), CRP monoclonal antibody (C7) (Biotin) and human CRP recombinant protein were purchased from Invitrogen (Carlsbad, CA, USA). Rat activin A beta A subunit antibody, rat activin A beta A subunit biotinylated antibody, recombinant activin A, rat VEGF164 antibody, rat VEGF biotinylated antibody, recombinant rat VEGF164 protein, rat CRP antibody, rat CRP biotinylated antibody, recombinant rat CRP protein were purchased from R\&D Systems (Minneapolis, MN, USA). Water was purified using a Millipore purification system (Burlington, MA, USA).

Device Design and Fabrication. All the plates were fabricated using digital light processing (DLP) 3D printing, which had a high printing resolution and allowed standardization of the microfluidic devices, with low manufacturing cost (less than $\$ 1$ in material cost per plate) and rapid fabrication process (5 minutes per plate). The printer we use was an Asiga Pico2 HD 3D printer, which has an LED peak wavelength of $385 \mathrm{~nm}$, an X-Y plane resolution (pixel size) of $37 \mu \mathrm{m}$ and a Z-axis control of $1 \mu \mathrm{m}$. 3D structures of the microfluidic devices were designed using SolidWorks and uploaded to the 3D printer. The detailed design of channel plates and well plates are demonstrated in Supplementary Figure S1. The printing material for the well plates was poly(ethylene glycol) diacrylate (PEGDA, MW 250) with 0.5\% (w/w) Irgacure 819. For channel plates, the printing material was PEGDA 250 with $0.5 \%$ (w/w) Irgacure 819 and $0.5 \%$ (w/w) nitrophenyl phenyl sulfide (NPS). NPS was used as an absorber to control the penetration depth of the incident light and enable the microchannels to be printed inside the chip. We set the build layer thickness as $50 \mu \mathrm{m}$, with the exposure time of each layer as $0.3 \mathrm{~s}$ for well plates and $1 \mathrm{~s}$ for channel plates. After the 3D printing was completed, the chips were immediately flushed with isopropanol to dissolve the unpolymerized material. The chips were then post-cured by exposure to UV light (365 
$\mathrm{nm}$ ) for $5 \mathrm{~min}$. To enhance the transparency of the device and enable the microscope observation, the printed chips were glued onto glass slides with epoxy glue.

A commercial superhydrophobic coating reagent, Ultra-Ever Dry, was applied to the outer surfaces of channel plates and well plates. An air powered sprayer was used to spray the two layers of coating reagents, generating uniform layers on the device surfaces. To avoid unwanted superhydrophobic coating on inner surfaces of the wells, we used 3D printing to fabricate shield modules (Figure S1c), which could assemble with the well plates and shelter the wells from the coating spray. The bottom coating was dried for $30 \mathrm{~min}$, and the top coating was dried for $2 \mathrm{~h}$. After the coatings were cured, all the devices were washed with water and dried by air flow.

Validation of the Loading, Incubation and Washing Process. Loading: Guided by the aligned structures on the four corners, miniaturized 96-well plates were assembled with channel plates and clamped to fix the position. Food colors, resorufin or Alexa 488-streptavidin (SA) $(10 \mu \mathrm{g} / \mathrm{mL})$ solutions were loaded into desired fluid paths by pipette injection from respective inlets. Since the system is not completely sealed, there are narrow interstices between plates, which allow air to escape and avoid forming bubbles. Fluid paths can also be partially filled by stopping the liquid injection at anywhere the users need, and the loaded liquid will not flow backward. After loading, the two plates were disassembled. Superhydrophobic coating facilitates to hold the liquid in their respective microchannels or wells during plate disassembly. Because both design and coating among wells are uniform, the volumes of liquids left in wells are consistent across the plate. For the current design, the volume of residual reagents left in channels for loading each well is as small as $0.8 \mu \mathrm{L}$. To evaluate the volume consistency, a fluorescence image of the whole well plate was taken by an imaging system (ChemiDoc MP imager, Bio-Rad). The fluorescence profiles along each lane were analyzed using the matched software.

Incubation: Horseradish peroxidase (HRP)-coated magnetic beads were prepared by suspending $2.9 \mu \mathrm{m}$ polystyrene (PS) magnetic beads in $10 \mu \mathrm{g} / \mathrm{mL}$ SA-HRP solution, incubating for $1 \mathrm{~h}$ and washing three times with PBS. For validation, one well plate was loaded with amplex red/ $\mathrm{H}_{2} \mathrm{O}_{2}$, and the other well plate was loaded with HRP-coated beads or uncoated beads in every other lane. The two plates were assembled face-to-face, with a magnet placed underneath the bead plate. The magnetic beads were then pulled into the wells with substrates by the magnet and incubated for 15 min for enzymatic reactions. After incubation, beads were pulled back into the bead plate by the magnet, followed by the separation of the two well plates. Fluorescence signals in each well of the substrate plate were then measured by using a fluorescence microscope (Olympus IX-73, Tokyo, Japan) with a sCMOS camera (Hamamatsu ORCA Flash 4.0V3 Lt).

Washing: Resorufin and Alexa-488 SA solutions were loaded into the well plate every other lane. The plate was put into a washing chamber, and the washing buffer (PBS with $0.1 \%$ Tween-20) was poured into the chamber until the whole plate was immersed. The chamber was shaken for different time periods ( $1 \mathrm{~min}, 3 \mathrm{~min}, 5 \mathrm{~min}$ ), and the buffer was then poured out. The plate was also washed 1, 2 or 3 times, each for 3 min duration. Fluorescence signals in each well under both green and red fluorescent channels were measured by a fluorescence microscope before and after every washing step.

Single Biomarker Analysis. $2 \mu \mathrm{L}$ of $2.9 \mu \mathrm{m}$ PS magnetic beads (2.5 wt \%) were first washed with PBS, suspended in $20 \mu \mathrm{L}$ IL- 6 capture antibody, and incubated for $2 \mathrm{~h}$ at room temperature. After coating, beads were 
washed three times with PBS, and blocked with General Block buffer for $30 \mathrm{~min}$. The beads were washed with PBS again. For parameter optimization, $0.5,0.25$, or $0.1 \mathrm{mg} / \mathrm{mL}$ of IL-6 capture antibody solutions were used for bead coatings; beads were resuspended in $4 \%$ BSA to give a final concentration of $0.25,0.1,0.05$, or $0.025 \mathrm{wt} \%$. During bead coating, four well plates were assembled with their channel plates and all loaded with General Block buffer. The plates were separated and blocked for $2 \mathrm{~h}$ in a humidified box. The plates were then dried by air flow and kept in a humidified environment until use. For establishing calibration curves of IL-6, serial dilution of IL-6 standards was performed with 4\% BSA to obtain different concentration standards $(1000,500,200,100,50,20$, $10,5,2,0 \mathrm{pg} / \mathrm{mL})$. Detection antibody and SA-HRP mixture were prepared by mixing IL-6 detection antibody with SA-HRP in $4 \%$ BSA to give both final concentrations of $2,1,0.5$ or $0.2 \mu \mathrm{g} / \mathrm{mL}$. Amplex red/ $\mathrm{H}_{2} \mathrm{O}_{2}$ substrate solution was prepared from the QuantaRed ${ }^{\mathrm{TM}}$ kit and used within $20 \mathrm{~min}$ after preparation.

For single biomarker analysis, capture antibody-coated magnetic beads, samples/standards, detection antibody and SA-HRP were preloaded into the bead plate, sample plate and antibody plate respectively in designed patterns. The sample plate was first assembled to the bead plate, which had a magnet underneath. After assembly, the two plates are fixed with adhesive tape. The magnetic beads were pulled into corresponding sample wells by a magnet for incubation. During the incubation, the beads were moved by the magnet every 5 min. After the incubation, magnetic beads were pulled back to the bead plate and the two plates were separated. The bead plate was then washed twice (each for $1 \mathrm{~min}$ ) and assembled with an empty well plate (well height: $0.25 \mathrm{~mm}$ ) with a magnet to remove extra solution from each well. The bead plate was then incubated with the antibody plate and washed four times (each for $1 \mathrm{~min}$ ) after incubation. During this process, substrate solution was loaded into the substrate plate. The bead plate was assembled with the substrate plate, incubated for $10 \mathrm{~min}$, and disassembled with a magnet. The fluorescence signals in substrate plate were measured using a fluorescence microscope.

Multiplexed Biomarker Analysis. Magnetic beads coated with IL-6, CRP, CEA, PSA capture antibodies, respectively, were prepared as above, with capture antibody concentration of $0.25 \mathrm{mg} / \mathrm{mL}$ and final bead concentration of $0.05 \mathrm{wt} \%$. For cross-reactivity analysis, solutions only containing IL-6 (20 pg/mL), CRP (2 $\mathrm{ng} / \mathrm{mL}), \mathrm{CEA}(500 \mathrm{pg} / \mathrm{mL})$ or PSA $(2 \mathrm{ng} / \mathrm{mL})$, four-protein mixture (each marker has the same concentration as the single protein solutions), and blank buffer were prepared. For establishing calibration curves, mix standards were prepared by mixing the four proteins in $4 \% \mathrm{BSA}$, and serially diluting into different concentrations. Detection antibodies and SA-HRP were $1 \mu \mathrm{g} / \mathrm{mL}$ for all the tests. Spiked serum samples were prepared by adding standard proteins into 5-fold diluted bovine serum.

Capture antibody-coated beads were vertically loaded into the bead plate, and each type of capture antibody occupied two columns. Standards or samples were loaded horizontally, each occupying one row. Detection antibody and SA-HRP mixtures were loaded vertically, each type in two columns corresponding to the pattern of beads (Figure S1d). The procedure of multiplexed biomarker analysis is the same as single marker analysis.

Rat plasma analysis. Female Sprague Dawley (SD) rats, utilized in previous experiments, were purchased from Hilltop Laboratories (Scottdale, Pennsylvania), and housed in an Assessment and Accreditation of Laboratory Animal Care (AAALAC) approved facility at West Virginia University (WVU) under a regulated temperature and 12:12h light-dark cycle. All procedures were approved by the Institutional Animal Care and Use 
Committee of West Virginia University (\#1602000621). Rats were randomly assigned to either the sham-control $(\mathrm{n}=4)$ or nanoTiO ${ }_{2}$-exposure $(\mathrm{n}=5)$ groups and whole-body inhalation exposures lasted for 6 days beginning after gestational day (GD) 10 to decrease animal stress as previously described. ${ }^{1}$ Briefly, nano- $\mathrm{TiO}_{2}$ aerosols were generated using a high-pressure acoustical generator (HPAG, IEStechno, Morgantown, West Virginia) to reach an average target concentration of $12 \mathrm{mg} / \mathrm{m}^{3}$. Sham-control animals were exposed to HEPA-filtered air only with similar temperature and humidity chamber conditions On GD 20, twenty-four hours after the last exposure, dams were anesthetized with $5 \%$ isoflurane and maintained on $2.5 \%$ isoflurane throughout surgery as previously described. ${ }^{2}$ Whole blood was collected from these rats via carotid artery cannula from living animals directly into tubes containing $5.4 \mathrm{mg} \mathrm{K} \mathrm{K}_{2}$ EDA (BD, Franklin Lakes, NJ) and immediately centrifuged for 10 minutes at 1100 $\times \mathrm{g}$. The plasma (supernatant) was then transferred into $1.5 \mathrm{~mL}$ tube and snap-frozen in liquid nitrogen before being placed at $-80^{\circ} \mathrm{C}$ for long-term storage.

Magnetic beads for activin detection were prepared by covalent modification of the activin capture antibody to the $1 \mu \mathrm{m}$ NHS-activated magnetic beads according to the protocol. VEGF and CRP beads were prepared by non-covalent coating as mentioned above. All the beads and well plates were blocked by the ChonBlock buffer, which demonstrated the best results for plasma sample analysis. Plasma samples were slow-thawed and placed on ice. For activin and VEGF analysis, plasma samples were diluted 5-fold in ChonBlock buffer. For CRP analysis, since the CRP was in a high original concentration, plasma samples were 40,000-fold diluted. The working procedure is similar to the multiplexed biomarker analysis. However, because of the large differences (about 100,000-fold) in original concentration between CRP and activin or VEGF, to avoid cross-contamination during washing, the bead plate was split into two parts. The loading and incubation processes were performed simultaneously for the three biomarkers, but the washing process was performed separately, with VEGF and activin sections washing together and CRP section washing alone.

(1) Bowdridge, E. C.; Abukabda, A. B.; Engles, K. J.; McBride, C. R.; Batchelor, T. P.; Goldsmith, W. T.; Garner, K. L.; Friend, S.; Nurkiewicz, T. R. Maternal Engineered Nanomaterial Inhalation During Gestation Disrupts Vascular Kisspeptin Reactivity. Toxicol. Sci. 2019, 169, 524-533.

(2) Abukabda, A. B.; Bowdridge, E. C.; McBride, C. R.; Batchelor, T. P.; Goldsmith, W. T.; Garner, K. L.; Friend, S.; Nurkiewicz, T. R. Maternal Titanium Dioxide Nanomaterial Inhalation Exposure Compromises Placental Hemodynamics. Toxicol Appl Pharmacol 2019, 367, 51-61. 